\title{
Application of BP Network based on GA in Student Evaluation
}

\author{
Shixin $\mathrm{Li}^{\mathrm{a}}$, Hai Zhang ${ }^{\mathrm{b}}$, Kaixin Li and Chaonan Fan \\ College of Electronic Engineering, Tianjin University of Technology and Education. 300222 Dagu \\ South Road, Tianjin, China \\ ali_shixin@sina.com, b1459399409@qq.com
}

\begin{abstract}
The examination evaluation of students is a complex, multi factor, multi variable nonlinear system. The traditional evaluation method is difficult and fair to reflect the students' learning effect. In this paper, the student examination evaluation system model based on the BP neural network based on GA is set up, the student sample results are collected according to the index, data mining technology is used for learning and training, and the trained model is applied to performance prediction. The simulation results show that the prediction accuracy of the student test evaluation model of the BP neural network optimized by GA is far more than the simple BP network prediction model and can overcome the interference of various subjective factors to the students' performance.
\end{abstract}

Keywords: Student examination evaluation, the BP neural network optimized by GA, Data mining technology.

\section{Introduction}

The exam evaluation of students is an important part of the education system. The exact result of the exam is directly related to the training of school talents. How to evaluate students' learning progress more accurately and rationally is an important subject in the process of deepening teaching reform and promoting quality education in an all-round way. However, in the actual work of student evaluation, there are many factors that affect the evaluation, and there may be correlation between the factors, which will make it difficult to express the results of student evaluation with a linear mathematical formula or a simple weighted average [1]. This paper collects the sample data according to the designed evaluation index and constructs the BP neural network student examination evaluation system model based on GA [2], at this time, we make full use of data mining technology to analyze and find the internal relationship among the students' performance indicators and accurately predict and assess. The weight, threshold and network parameters of BP network is optimized and adjusted by genetic algorithm, so that the network error tends to be minimal, and the more accurate evaluation results are obtained.

\section{Introduction of BP Neural Network based on GA}

In recent years, the theory and application of artificial intelligence have been developed rapidly. Genetic algorithm (GA) and neural network are two important branches. Both of them apply bionics theory to scientific research. They can solve nonlinear and uncertain problems better. The combination of the two methods can have both the wide mapping ability of neural network and the fast and global convergence of GA. It can effectively avoid the shortcomings of slow convergence speed and easy to be limited to local minimum of traditional neural network learning algorithm. BP neural network based on GA uses GA to optimize the weights of the neural network, locates some better search space in the solution space, and then uses BP algorithm to search the optimal solution in these solution spaces [3]. In this paper, GA is used to optimize the connection weights and thresholds of BP neural network model to improve its training speed and overcome its shortcomings of easily falling into local minimum. 


\section{Student Examination Evaluation Model based on BP Neural Network Optimized by GA}

\subsection{Construction of BP Neural Network Model Optimized by GA.}

BP neural network is very sensitive to the initial weights of the network because of its gradient descent learning and training method. Different weights can cause a great variety of network outputs [4]. In the selection of parameters such as learning rate and activation function, because there is often no clear theoretical basis, it is determined entirely according to empirical formula. If it is not properly selected, it will lead to a series of problems, such as poor network stability, local optimization of network output, long network training time and so on, which will ultimately affect the generalization ability of the network [5,6]. Genetic algorithm has the ability of global search, and the search range includes the whole solution space, which is conducive to the search of global optimal solution [7]. In this paper, genetic algorithm is used to optimize the weight of BP neural network to speed up the convergence time and avoid the network falling into local optimum [8]. The optimization process of network weight is as follows:

(1) The mixed coding method is used to encode the network weights, and a distribution is generated arbitrarily, which corresponds to a set of network connection weights.

(2) Calculate its error function through the input network learning data. The fitness is inversely proportional to the error, the higher the fitness, the smaller the network error, so as to judge the network weight.

(3) Through selection operation, the individuals with higher fitness in the population were selected to inherit the next generation.

(4) Through crossover operation, we can find out the individuals with higher fitness to the next generation on the basis of selection operation.

(5) Gene coding of some individuals is changed by mutation operation to ensure individual diversity and produce the next generation of population.

(6) Repeat steps (2) to (5) so that the initial generated network weight is constantly updated until the network stopping condition is met [9].

After the normalized original sample data is encoded by the chromosome of genetic algorithm, the individual fitness is judged, and the operations of selection, crossover and mutation are repeated until the optimal solution of the population is found. Among them, the number of initial populations is 250 , and the hereditary algebra is 100 . After population evolution, the weights and thresholds of BP network are optimized, and the initial weights and thresholds are assigned to the BP network which has not yet started training.

net. IW $\{1,1\}=W 1$; net. $\operatorname{LW}\{2,1\}=W 2$; net. $\mathrm{b}\{1\}=\mathrm{B} 1$; net. $\mathrm{b}\{2\}=\mathrm{B} 2$;

Net. IW $\{1,1\}$ is the weight between the input layer and the hidden layer, net. $\operatorname{LW}\{2,1\}$ is the weight between the hidden layer and the output layer, net. $b\{1\}$ is the threshold between the input layer and the hidden layer, and net. $\mathrm{b}\{2\}$ is the threshold between the hidden layer and the output layer.

\subsection{Construction of Evaluation Index System.}

Based on the principles of systematisms, pertinence, practicality and independence, the author takes his own school as the research object to analyze the factors influencing students' learning effect and the degree of influence of each factor. Referring to the system standards of other brothers' colleges and universities, this paper chooses four first-level indicators of classroom performance, extension and development, professional practice and performance evaluation, and 14 second-level indicators of classroom attendance and classroom discipline to construct the evaluation index system, and distributes the score reasonably according to the influence degree of the indicators on students' performance. 


\subsection{Acquisition of E-learning Samples.}

This paper adopts supervised learning. Sample data is the teacher of network learning, the quality of training samples directly determines the quality of network model, so the collection of training samples should be paid special attention. In the index, the scores of classroom performance, professional practice and so on are obtained by the teacher through the usual records and performance appraisal records. Each teacher should give the most real feedback to the students' actual performance. Under the supervision and guidance of the head teacher, the evaluation group is composed of the monitor, the study committee and the students' democratic recommendation representatives. The evaluation team should collect all the materials related to the content of the index, evaluate the students objectively and impartially and score them independently, and finally get the input data of the index.

\subsection{Acquisition of Sample Data.}

In this paper, according to the requirements of indicators, a course of 30 students in a class of electronic information specialty was tracked for one semester. The collected sample data are shown in Table 1. Students' grades are strictly accounted for in the table according to the ratio of indicators: if the final grade is 89 , it will be accounted for in accordance with $89 \times 70 \% \approx 62$. Experts score is the result of each sample data calculated according to the index system proportion. The first 25 sets of data in the form are used as learning data for training the learning network model. The latter 5 sets of data are test samples, which are used to compare training errors to determine the performance of the obtained network models.

Table 1. Sample Data table

\begin{tabular}{cccccccccc}
\hline & 1 & 2 & 3 & 4 & $\ldots$ & 27 & 28 & 29 & 30 \\
\hline Attendance & 20 & 18 & 14 & 19 & $\ldots$ & 20 & 13 & 19 & 17 \\
Classroom discipline & 19 & 18 & 16 & 18 & $\ldots$ & 20 & 18 & 15 & 17 \\
Classroom assignments & 37 & 37 & 32 & 28 & $\ldots$ & 29 & 20 & 27 & 27 \\
Classroom interaction & 12 & 16 & 12 & 13 & $\ldots$ & 14 & 14 & 19 & 18 \\
Outside & 8 & 9 & 8 & 9 & $\ldots$ & 10 & 5 & 8 & 9 \\
reading & $\ldots$ & $\ldots$ & $\ldots$ & $\ldots$ & $\ldots$ & $\ldots$ & $\ldots$ & $\ldots$ & $\ldots$ \\
$\ldots$ & 20 & 19 & 13 & 20 & $\ldots$ & 20 & 18 & 19 & 20 \\
Field test performance & 58 & 55 & 42 & 52 & $\ldots$ & 56 & 34 & 58 & 46 \\
Final & & & & & & & & & \\
exam & 90 & 87 & 72 & 84 & $\ldots$ & 92 & 65 & 88 & 81 \\
Expert & & & & & & & &
\end{tabular}

\subsection{Training Results and Analysis.}

In order to verify the superiority of the BP neural network algorithm optimized by GA, the prediction accuracy of the two algorithms is compared. Table 2 is a comparison of errors between expert scoring and test results. It can be seen that the prediction accuracy of GA-BP neural network is much better than that of BP neural network. The BP neural network evaluation method based on GA can greatly reduce the evaluation time and better overcome the subjective factors, and enhance the scientific, effectiveness and rationality of students' evaluation results.

Table 2. Error comparison between Expert scoring and Test results

\begin{tabular}{ccc}
\hline Numble & GA-BP & BP \\
\hline 26 & 0.071761 & 1.0542 \\
27 & 0.047872 & 0.5673 \\
28 & 0.063983 & 0.84646 \\
29 & 0.067602 & 1.9414 \\
\hline
\end{tabular}


In addition, the convergence speed of GA-optimized BP neural network is obviously faster than that of BP neural network alone. As can be seen from Figures, for GA-BP neural network, when the number of training steps is 176 , the error value is $10-6$, to achieve learning accuracy, the error is basically stable. For BP neural network to achieve the same accuracy, 187 steps are needed.

The convergence curve of population evolution of genetic algorithm is shown in Figure 1. It can be seen that when the genetic evolution reaches the 30 generation, the population evolves to the optimal state.

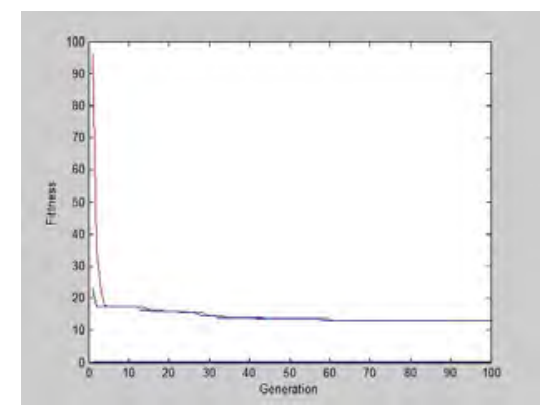

Fig. 1 The convergence curve of population evolution of genetic algorithm

\section{Summary}

In this study, an unsupervised learning method is proposed to optimize the BP neural network evaluation model through GA to train and process the students' performance data. The result of BP neural network training is compared with that of BP neural network optimized by genetic algorithm. The result shows that the optimized BP neural network has better convergence performance, greatly improved prediction accuracy and minimal error. It can provide a new and effective evaluation method for vocational education evaluation. In practical application, the model can also be improved according to the specific requirements of the evaluation, so as to replace the manual for more complex evaluation activities.

\section{References}

[1]. Guangyang Huang. Education measurement and evaluation[M]. Shanghai: East China Normal University Press,2012.

[2]. Juping Peng. The research of the application of the BP neural network in teaching quality evaluation for national college[D]. Gansu: Lanzhou University,2013.

[3]. Zhang Zonghua, Zhao Jingxiang, Lu Heng, Niu Xinzheng. Application of BP neural network based on Genetic Algorithm in power load forecasting [J]. Computer engineering,2017,43 (10): $277-282+288$.

[4]. Ming Chen. The principle and practical example of MATLAB neural network[M]. Beijing: Tsinghua university press, 2013.

[5]. Defeng Zhang. MATLAB Neural Network Programming [M]. Beijing: Chemical Industry Press, 2011.

[6]. Wuqiang, Tong Xuefeng, Ji Jun. Digital character recognition based on artificial neural network [J]. computer engineering.2003(14):112-113+132.

[7]. Lei Yingjie, Zhang Shanwen, Li Xuwu, et al. Genetic algorithm toolbox and its application [M]. Xi'an: Xi'an University of Electronic Science and Technology Press,2005:45-60. 
[8]. Montana D J, Davis L. Training Feed Forward Neural Networks Using Neural Networks and Genetic Algorithm[C]/Proc. of International Conference on Computing, Communications and Control Technologies. Austin, USA: [s. n.], 1989: 762-767.

[9]. Li Weihong, Chen Yebin, Wen Lei. Dengue space-time diffusion simulation based on GA-BP neural network model [J].2015,20(7): 0981-0991. 